NBER WORKING PAPER SERIES

\title{
PRICE STICKINESS IN SS MODELS: NEW INTERPRETATIONS OF OLD RESULTS
}

\author{
Ricardo J. Caballero \\ Eduardo M.R.A. Engel \\ Working Paper 12945 \\ http://www.nber.org/papers/w12945
NATIONAL BUREAU OF ECONOMIC RESEARCH
1050 Massachusetts Avenue
Cambridge, MA 02138
March 2007

\begin{abstract}
Respectively: caball@ mit.edu; eduardo.engel@yale.edu. We thank Ruediger Bachmann, Olivier Blanchard,Bill Brainard, Mike Golosov, Oleksy Kryvtsov, John Leahy, Virgiliu Midrigan, and participants at the Conference on "Microeconomic Adjustment and Macroeconomic Dynamics" (Study Center Gerzensee, October 20, 2006); the New York Workshop on Monetary Economics (NY Fed, November 17, 2006), and the Duke macroeconomics workshop for helpful comments on an earlier draft of this paper (September, 2006) titled "Price Stickiness in Ss Models: Basic Properties." The authors thank the NSF for financial support. The views expressed herein are those of the author(s) and do not necessarily reflect the views of the National Bureau of Economic Research.

(C) 2007 by Ricardo J. Caballero and Eduardo M.R.A. Engel. All rights reserved. Short sections of text, not to exceed two paragraphs, may be quoted without explicit permission provided that full credit, including () notice, is given to the source.
\end{abstract}


Price Stickiness in Ss Models: New Interpretations of Old Results

Ricardo J. Caballero and Eduardo M.R.A. Engel

NBER Working Paper No. 12945

March 2007

JEL No. E32,E62

\begin{abstract}
$\underline{\text { ABSTRACT }}$
What is the relation between infrequent price adjustment and the dynamic response of the aggregate price level to monetary shocks? The answer to this question ranges from a one-to-one link (Calvo, 1983) to no connection whatsoever (Caplin and Spulber, 1987). The purpose of this paper is to provide a unified framework to understand the mechanisms behind this wide range of results. In doing so, we propose new interpretations of key results in this area, which in turn suggest the kind of Ss model that is likely to generate substantial price rigidity. The first result we revisit is Caplin and Spulber's monetary neutrality model. We show that when price stickiness is measured in terms of the impulse response function, this result is not a consequence of aggregation, but is due instead to the absence of price-stickiness at the microeconomic level. We also show that the "selection effect," according to which units that adjust their prices are those that benefit most, is neither necessary nor sufficient to account for the higher aggregate flexibility of Ss-type models compared to Calvo models. Instead, the key concept is the contribution of the extensive margin of adjustment to the aggregate price response. The aggregate price level is more flexible than suggested by the microeconomic frequency of adjustment if and only if this term is positive.

Ricardo J. Caballero

Department of Economics

MIT

Room E52-252a

Cambridge, MA 02142-1347

and NBER

caball@mit.edu

Eduardo M.R.A. Engel

Yale University

Department of Economics

P.O. Box 208268

New Haven, CT 06520-8268

and NBER

eduardo.engel@yale.edu
\end{abstract}




\section{Introduction}

Understanding the response of the aggregate price level to monetary shocks is among the central questions in monetary economics. Since the origin of almost any aggregate nominal rigidity is a microeconomic rigidity, there have been many studies documenting microeconomic pricing behavior, in particular the frequency of microeconomic price adjustment. ${ }^{2}$

For these studies to be relevant for macroeconomic policy, we need to understand the mapping from infrequent price adjustment to aggregate price stickiness. We already know that this mapping can be surprising. Caplin and Spulber (1987), henceforth CS, construct an insightful example where there is no relation between these two measures. They combine a one-sided $S s$ model of microeconomic price adjustment with a specific form of asynchronous adjustment of individual prices (the assumption of a uniform cross-section), and obtain an aggregate price level that responds one-for-one to monetary shocks. Thus there is no aggregate price stickiness in their model - the impulse response is one upon impact and zero thereafter - despite the fact that the frequency of microeconomic price adjustments can take any value.

In a related recent result, Golosov and Lucas (2006) show that the sluggishness of the aggregate price response to monetary shocks is overestimated when approximating a menu-cost model with a Calvo model, where adjustment is infrequent but uncorrelated with the size of price imbalances. That is, the frequency of microeconomic price adjustments underestimates the flexibility of the aggregate price level in Ss models. Similarly, Bils and Klenow (2004) report that the flexibility of aggregated price series in U.S. retail data is significantly higher than suggested by the frequency of price adjustments observed in microeconomic data: they estimate a median monthly frequency of price adjustments of 0.21 , while one minus the firstorder autocorrelation of the aggregate inflation series - a natural measure of aggregate price flexibility which in their Calvo setting should equal the adjustment frequency - is almost four times as large.

Aside from these illustrative examples, is there anything more general that can be said about the connection between the frequency of microeconomic adjustment and the degree of flexibility of the aggregate price level? What are the precise channels through which microeconomic inaction and aggregate price stickiness are connected in Ss models? The purpose of this paper is to answer these questions and, along the way, offer new interpretations for some old results.

\footnotetext{
${ }^{2}$ See Bils and Klenow (2004), and Nakamura and Steinsson (2006a) for the U.S., and Dhyne et al. (2006) and Fabiani et al. (2006) for a summary of the findings of the impressive set of country studies sponsored by the ECB's Inflation Persistence Network.
} 
There are two new interpretations and mechanisms we discuss in this paper that stand out. First, the main result in CS is that in their context a small monetary expansion has no effect on aggregate output, despite the fact that at any given instant most microeconomic units do not adjust their prices. The standard interpretation of this result is that aggregation erases the impact of microeconomic stickiness. Our first point is that this interpretation depends crucially on the concept of price stickiness being used. If, at the micro level, this concept is defined in terms of the frequency of price adjustments, then the statement is correct, since money is neutral at the aggregate level despite the fact that most firms do not adjust their prices at the micro level. Yet if we have in mind a more standard definition of stickiness, in terms of the impulse response to monetary shocks, then the usual interpretation of Caplin and Spulber's money neutrality result changes dramatically, as now it reflects the absence of microeconomic stickiness.

Second, Golosov and Lucas (2006) coined the expression "selection effect" to describe the feature of $S s$ type models that adjusting firms are those that need it most, in sharp contrast with Calvo's model, where adjustment is completely random. They argue informally, and others have followed, that this feature explains the higher aggregate flexibility of $S s$ type models over Calvo models, for a given frequency of microeconomic adjustment. We show that while the selection effect is present in most $S s$ type models, it is not the key concept behind their additional flexibility.

Instead, the key concept is that of an extensive margin. That is, of the additional price increase resulting from the rise in the fraction of agents adjusting upwards and the fall in the fraction of agents adjusting downwards, both as a consequence of an additional monetary impulse. It contrasts with the intensive margin, which describes the additional price increase (or reduced price decrease) of those firms that were going to adjust anyway. Only the intensive margin is active in the Calvo model, while in $S s$ type models both margins are strictly positive. This extensive margin is a generalization of the effect of the number of agents reaching the trigger thresholds in $S s$ models discussed by Bar-Ilan and Blinder (1992)..$^{3}$

Section 2 revisits the Caplin and Spulber model. Section 3 begins our study of the relation of aggregate price flexibility and the frequency of microeconomic adjustment, considering a simple extension of the Caplin and Spulber model that includes the Calvo model as a limiting case. Sections 4 and 5 are the core of the paper: they describe the key results in the context

\footnotetext{
${ }^{3}$ Incidentally, while Golosov and Lucas (2006) used the expression "selection effect" to describe the mechanism behind their finding, they do point out in their formal analysis that the key difference between the Calvo model and their particular $S s$ model is the change in the number of agents that adjust in response to a monetary impulse. The point, however, is that selection and extensive margin are not synonyms.
} 
of a generalized $S s$ model, which includes standard $S s$ models as a particular case. Section 6 concludes and is followed by an appendix.

\section{Caplin and Spulber Revisited}

This section recreates and clarifies the source of the Caplin and Spulber monetary neutrality result. It also provides a natural starting point for the topics we cover in later sections.

\subsection{The Model}

Let us focus on the aspects of the model which are relevant to our concerns, skipping the derivation of the underlying microeconomic rules or a discussion of general equilibrium aspects, which are largely orthogonal to the issues we address (see, e.g., Stokey (2002) and Dotsey, King and Wolman (1999) for useful references on the steps we skip).

There exists a continuum of firms indexed by $i \in[0,1]$. Let $p_{i t}$ and $p_{i t}^{*}$ denote the $(\log$ of the - henceforth omitted) actual and target price, respectively, both for firm $i$ at time $t .{ }^{4}$ In CS there are no idiosyncratic shocks and, leaving aside inessential constants:

$$
p_{i t}^{*}=m_{t}
$$

where $m_{t}$ denotes the money stock. The sample paths of $m_{t}$ are continuous and increasing.

Aggregate output, $y_{t}$, is proportional to (the log of) real balances:

$$
y_{t}=m_{t}-p_{t}
$$

with the aggregate price level, $p_{t}$, defined as

$$
p_{t} \equiv \int p_{i t} d i
$$

If there are no frictions in microeconomic price adjustment, $p_{i t}=p_{i t}^{*}=m_{t}$, so that $p_{t}=m_{t}$ and money is neutral. Suppose instead that there is a fixed cost of adjusting individual prices and hence firms adopt $S s$ rules in setting their prices. As usual, it is convenient to define a state variable:

$$
x_{i t} \equiv p_{i t}-p_{i t}^{*}
$$

\footnotetext{
${ }^{4}$ In a model with stochastic adjustment costs, $p_{i t}^{*}$ is defined formally as the price the agent would choose, conditional on the current state of the economy, if its current adjustment cost draw is equal to zero.
} 
The adjustment rule is such that when $x_{i t}$ reaches $s-S$, the firm increases the price by $S-s$. This large adjustment catches up with the accumulated monetary expansion since the previous adjustment and anticipates some of the expansion that will take place before the next adjustment (recall that $p_{i t}^{*}$ is equal to $m_{t}$ plus a constant; furthermore, following the convention used in generalized $S s$ models, this constant is chosen so that the price imbalance $x$ is zero immediately after firms adjust their prices).

In CS, firms' adjustments are not perfectly synchronized because the initial cross-section distribution of actual prices is non-degenerate. In particular, CS assume that the initial distribution of $x$ is uniform over the entire $(s-S, 0]$ interval. It turns out that under the monotonicity and continuity assumptions for the sample paths of money, this uniform distribution is invariant: While the position of individual firms in state space changes over time, the cross-section distribution remains unchanged and uniform over $(s-S, 0]$. Figure 1 , taken from CS, illustrates the variation over time of $r \equiv x+S$, for an agent $i$; note that in the absence of idiosyncratic shocks, the distance between agents on the circle remains unchanged.

Figure 1: The Caplin and Spulber Model

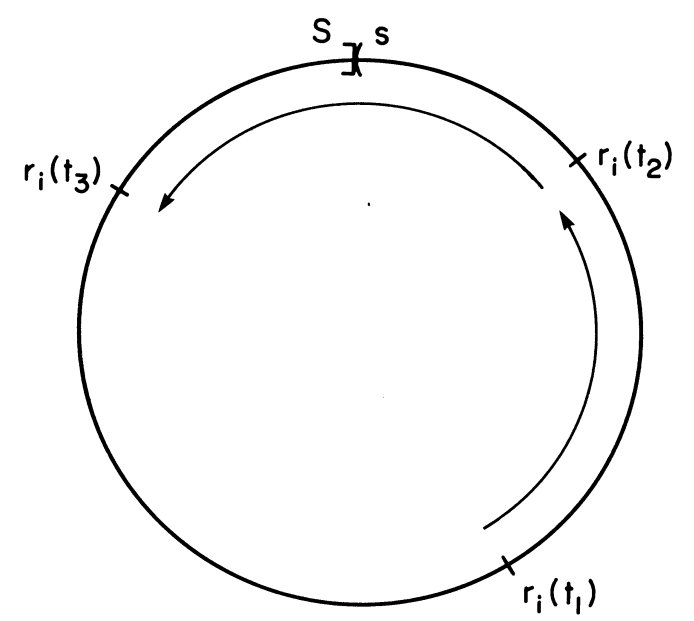

\subsection{Main Result}

The main result in CS is that in this context a small monetary expansion has no effect on aggregate output, despite the fact that at any given instant most microeconomic units do not adjust their prices. To see this result, note that a monetary expansion of $\Delta m$ triggers 
the adjustment of $\Delta m /(S-s)$ firms, and each of these firms increases its price by $(S-s)$. The change in the aggregate price level is simply the product of these two terms:

$$
\Delta p=\frac{\Delta m}{S-s} \times(S-s)=\Delta m
$$

and hence

$$
\Delta y=0
$$

The standard interpretation of this result is that aggregation erases the impact of microeconomic stickiness. Our first point is that this interpretation depends crucially on the concept of price stickiness one has in mind. If this concept is defined in terms of the frequency of microeconomic price adjustments, then the statement is correct, since money is neutral at the aggregate level despite the fact that most firms do not adjust their prices at the micro level. Yet if one has in mind a more standard definition of stickiness, in terms of the impulse response to monetary shocks, then the usual interpretation of Caplin and Spulber's money neutrality result changes, as now it reflects the absence of microeconomic stickiness.

We support our claim in two steps. Consider first the price-response $\Delta p_{i}(\Delta m, x)$ of a representative firm $i$, with state variable $x$ to a small monetary shock of size $\Delta m$. A shift of $x$ by $\Delta m$ leads to no adjustment if the firm is at a distance larger than $\Delta m$ from the trigger barrier. Only if $x$ is close enough to $s-S$ does the firm adjust, from (approximately) $s-S$ to $0 .^{5}$

Therefore:

$$
\Delta p_{i}(\Delta m, x)= \begin{cases}0, & \text { if } x>s-S+\Delta m \\ S-s, & \text { otherwise }\end{cases}
$$

and it follows that:

$$
\frac{\Delta p_{i}(\Delta m, x)}{\Delta m}= \begin{cases}0, & \text { if } x>s-S+\Delta m \\ (S-s) / \Delta m, & \text { otherwise }\end{cases}
$$

To obtain a measure of microeconomic flexibility in terms of the impulse response function, we average this expression over all possible values of $x$. The obvious candidate to weigh the different values of $x$ is the average time-distribution (ergodic distribution) of the state

\footnotetext{
${ }^{5}$ Strictly speaking, the adjustment is from $x-\Delta m$ to 0 , but $s-S-x+\Delta m$ is sufficiently small for adjusting firms that $s-S$ is a good approximation and simplifies the expressions. Of course, the limit as $\Delta m \rightarrow 0$ does not depend on this approximation.
} 
variable $x$ for a given firm. Denoting this density by $h_{E}(x)$ we have:

$$
\frac{\Delta p_{i}}{\Delta m} \equiv \int_{s-S}^{0} \frac{\Delta p_{i}(\Delta m, x)}{\Delta m} h_{E}(x) d x
$$

It is intuitively obvious (for a proof see Section 3) that a firm's ergodic density in CS is uniform on $(s-S, 0]$, so that $h_{E}(x)$ is constant and equal to $1 /(S-s)$.

The impulse response of $S s$ (and Calvo) type models typically decreases monotonically and adds up to one because of long run neutrality. Thus, much of the persistence of the IRF to monetary shocks is summarized by the initial response to such a shock. We therefore define our main measure of microeconomic price flexibility as:

$$
\mathcal{F}^{\text {micro }} \equiv \frac{\Delta p_{i}}{\Delta m}
$$

It follows that there is no price stickiness at the microeconomic level since:

$$
\mathcal{F}^{\mathrm{CS}, \text { micro }}=\int_{s-S}^{s-S+\Delta m} \frac{S-s}{\Delta m} \times \frac{1}{S-s} d x=1 .
$$

In other words, if we follow a firm over time and draw the histogram of its marginal responses to a monetary shock, $\Delta p_{i t} / \Delta m$, most of the observations pile up at zero. Yet a small fraction of observations pile up at $(S-s) / \Delta m$, corresponding to times where the response is much larger than one-for-one. The average value over time of $\Delta p_{i t} / \Delta m$,

$$
\frac{1}{T} \sum_{t=1}^{T} \frac{\Delta p_{i t}}{\Delta m}
$$

converges to one as $T$ tends to infinity.

\section{Property 1 (The IRF in Caplin-Spulber has no microeconomic price-stickiness)} In Caplin and Spulber: $\mathcal{F}^{\mathrm{CS} \text {,micro }}=1$.

It is easy to extend the above result to the complete impulse response function, beyond its first element. Denote by $\operatorname{IRF}_{k}^{\text {micro }}$ the average price response of a firm at time $k$ to a small monetary shock $\Delta m$ in period zero, normalized by the size of the shock. We then have:

$$
\mathrm{IRF}_{k}^{\mathrm{CS}, \text { micro }}=\left\{\begin{array}{cc}
1, & \text { for } k=0, \\
0, & \text { for } k \geq 1
\end{array}\right.
$$


Thus, not only $\mathcal{F}^{\text {micro }}$, but any reasonable definition of price-flexibility based on the entire IRF, assigns no microeconomic stickiness in the CS context.

The second step in our study of the IRF in Caplin and Spulber connects microeconomic and aggregate price stickiness. It follows from our derivation of Caplin and Spulber's resultsee equation (2) - that the aggregate response of the price level to monetary shocks is also one-for-one upon impact and zero thereafter. This is the well known CS result. Our point, however, is that the actual cross-section distribution has little to do with this result. Once the model has no microeconomic stickiness, the macroeconomic result follows regardless of what the cross-section distributions look like. We turn to this issue next.

\subsection{Relation between Micro and Macro Stickiness}

The result that micro and macro stickiness are the same is quite general and follows from the Ergodic Theorem (see, e.g., Walters (1982). We sketch the proof of this result next.

Given a cross-section $f(x)$ for the state variable, we have that the aggregate price response to a monetary shock $\Delta m$ can be defined as:

$$
\mathcal{F}(f) \equiv \int \frac{\Delta p_{i}(\Delta m, x)}{\Delta m} f(x) d x
$$

To obtain an aggregate measure of price flexibility, we need to average the above expression over all possible cross-sections $f(x): f_{1}(x), f_{2}(x), \ldots, f_{n}(x) .{ }^{6}$ The measure of aggregate price flexibility analogous to $\mathcal{F}^{\text {micro }}$ is therefore defined as:

$$
\mathcal{F}^{\text {macro }} \equiv \sum_{k=1}^{n} w_{k} \mathcal{F}\left(f_{k}\right)
$$

where $w_{k}$ denotes the weight of the $k$-th cross section, with $w_{k}>0$ and $\sum_{k=1}^{n} w_{k}=1$. Denoting the weighted average of all cross-sections by $f_{A}(x)$, and noting that the operator defined in (4) is linear, we have:

$$
\mathcal{F}^{\text {macro }}=\mathcal{F}\left(f_{A}\right)=\int \frac{\Delta p_{i}(\Delta m, x)}{\Delta m} f_{A}(x) d x .
$$

Of course, no averaging is needed for the CS model, since all cross sections $f_{k}(x)$ in $(5)$ are the same in this case (and uniform on $(s-S, 0]$ ). More generally, however, such an average

\footnotetext{
${ }^{6}$ The actual number of cross-sections is infinite and not countable, thus measure theory is required for a formal statement and proof. We assume a finite number for illustrative purposes.
} 
exists under rather weak conditions and, by the Ergodic Theorem, is equal to the individual firm's ergodic density, $h_{E}(x)$, considered in (3). Since $f_{A}(x)=h_{E}(x)$, comparing (3) and (6) yields the following property:

\section{Property 2 (Macro and micro price flexibility are always the same)}

Macro- and microeconomic price flexibility, as measured by $\mathcal{F}^{\text {macro }}$ and $\mathcal{F}^{\text {micro }}$, are the same in any (stationary) macroeconomic model:

$$
\mathcal{F}^{\text {macro }}=\mathcal{F}^{\text {micro }}
$$

Furthermore, a straightforward extension of the derivation of this result shows that the macro and micro impulse response to a monetary shock are the same at all lags. (Where the macro response is obtained by averaging over all cross sections, and the micro response by averaging over all individual time-series.)

Our interpretation claim for the CS model now follows from Properties 1 and 2:

\section{Property 3 (The source of aggregate price flexibility in Caplin and Spulber)}

When price stickiness is defined in terms of the impulse response function, the source of aggregate price flexibility in Caplin and Spulber is the absence of stickiness at the microeconomic level.

\subsection{The Role of the Cross-Section}

It is apparent from the previous property that the uniform cross-section distribution in CS has nothing to do with the absence of aggregate price stickiness in their model. So what is the role played by this assumption? The answer is that by choosing a distribution that does not vary over time, CS ensured that the response of the economy to aggregate shocks is the same at all moments in time. For most $S s$-type models this is not the case, since the cross-section varies endogenously over time, and so does any reasonable measure of price flexibility (for example, the one defined in (4)). To illustrate this point, we develop a simple generalization of CS (see Caballero and Engel (1991) for more details).

Consider the CS setting, except for the initial distribution of firms' state variable, which now covers only half the inaction range: the $S s$ bands are normalized to $S-s=2$ and the initial distribution is uniform on $[-3 / 2,-1 / 2]$. 
Figure 2: $p$ and $m$ in an extension of Caplin-Spulber

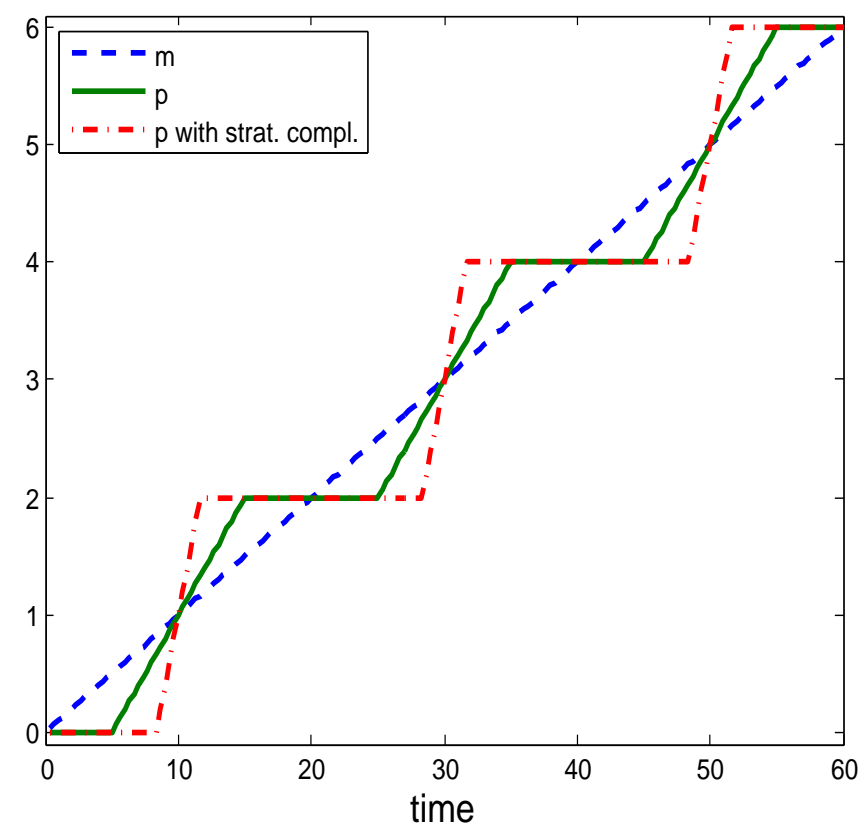

The solid line in Figure 2 depicts the evolution of the price level under the additional assumption that $m$ grows linearly over time at the rate $\mu$. Initially, there is a period where no firm adjusts its price and the aggregate price level does not change. Eventually, firms reach the trigger level $s-S$ and the aggregate price level rises twice as fast as money. After the last firm adjusts, a new period without price adjustment begins, and so on.

We first note that the ergodic density for a single firm continues to be uniform on the entire inaction range, $(-2,0]$. Thus our measure of micro price-flexibility, $\mathcal{F}^{\text {micro }}$, is equal to one as in the standard version of Caplin and Spulber, which from Property 2 implies that $\mathcal{F}^{\text {macro }}=1$ as well.

Since in this case $f$ varies over time, so does the conditional price-flexibility measure defined in (4). In this example it only takes two values, each one of them half the time:

$$
\mathcal{F}(f)=\left\{\begin{array}{l}
0 \quad \text { when no firm is adjusting }\left(f\left(-2^{+}\right)=0\right) \\
2 \text { when some firms are adjusting }\left(f\left(-2^{+}\right)=1\right)
\end{array}\right.
$$

Thus, what is special about CS within the class of one-sided $S s$ models is not that there is no aggregate price stickiness. It follows from the Average Neutrality Result in Caballero and Engel (1993a) that this is the case for a broad family of models of this type. Instead, what 
is special about CS's cross-section distribution is that it is invariant and hence the response of the aggregate price level to monetary shocks does not vary over time.

\subsection{Strategic Complementarities}

Let us conclude this section with an extension that incorporates strategic complementarities. Equation (1) becomes:

$$
p_{i t}^{*}=(1-a) m_{t}+a p_{t},
$$

where the parameter $a \in[0,1 / 2)$ captures the extent to which firms wish to coordinate their prices. Firms have stronger incentives to keep their prices in line with those of other firms when $a$ is larger.

The aggregate change in prices during a small time unit $\Delta t$ is given by:

$$
\Delta p=\Delta p^{*} f(s-S) \Delta t \times(S-s)
$$

where $\Delta p^{*} f(s-S) \Delta t$ is the fraction of firms that adjust and $S-s$ the size of their adjustments. Equation (8) extends (2) to this more general setting.

Substituting (7) in (8) and solving for $\Delta p$ leads to:

$$
\Delta p= \begin{cases}0 & \text { when no firm is adjusting }\left(f\left(-2^{+}\right)=0\right), \\ (2-2 a) \mu \Delta t /(1-2 a) & \text { when some firm are adjusting }\left(f\left(-2^{+}\right)=1\right) .\end{cases}
$$

Hence:

$$
\mathcal{F}(f)= \begin{cases}0 & \text { when no firm is adjusting }\left(f\left(-2^{+}\right)=0\right), \\ (2-2 a) /(1-2 a) & \text { when some firms are adjusting }\left(f\left(-2^{+}\right)=1\right) .\end{cases}
$$

The dash-dotted line in Figure 2 depicts the evolution of the price level when $a=0.4$. Compared with the case without strategic complementarities ( $a=0$, solid line), the aggregate price level remains constant during longer periods of time. The flip side is that when the aggregate price level increases, it does so at a faster rate, since a larger fraction of firms adjust their price in any given time period. The longer periods of inaction and the shorter but brisker periods with price adjustments cancel each other out so that the flexibility index $\mathcal{F}$ - which is equal to the average of $\mathcal{F}(f)$ - continues to be equal to one. 


\section{From Caplin-Spulber to Calvo}

What relation should we expect between the price adjustment frequency and aggregate price flexibility in menu-cost models? The one-sided $S s$ models described earlier, with complete price flexibility at the aggregate level regardless of the adjustment frequency, suggest that the answer is none. At the other extreme is the Calvo (1983) model, where both concepts coincide and aggregate flexibility is equal to the adjustment frequency. More generally, however, the answer is in between these two extremes.

In the CS model, the fraction of firms that adjust in one time period, henceforth the frequency of adjustment index, is equal to

$$
\mathcal{A}^{\mathrm{CS}}=\int_{s-S}^{s-S+\mu} \frac{1}{S-s} d x=\frac{\mu}{S-s}<1,
$$

where $\mu$, as before, denotes the money growth rate (assumed constant), $(s-S, 0]$ the inaction range, and we have assumed that the choice of time unit is such that there are always firms that do not adjust within a given period (i.e., $\mu<S-s$ ).

Since the price flexibility index $\mathcal{F}^{\mathrm{CS}}$ is one, we have that:

$$
\mathcal{F}^{\mathrm{CS}}>\mathcal{A}^{\mathrm{CS}}
$$

Let us now modify the one-sided $S s$ model and assume that in addition to the trigger threshold $s-S$, there is a strictly positive hazard $\lambda$ that a firm adjusts at any point in time, regardless of its price imbalance $x$. Thus, we have a model that nests both Calvo and one-sided $S s$ models: As $s$ tends to minus infinity we obtain the Calvo model, while if $\lambda=0$ we are back to CS.

If $f(x, t)$ denotes the cross-section density at time $t$, then

$$
f(x, t+\Delta t)=(1-\lambda \Delta t) f(x+\mu \Delta t, t), \quad s-S<x \leq 0 .
$$

This follows from the fact that a firm with a price imbalance $x$ at time $t+\Delta t$ must have had a price imbalance $x+\mu \Delta t$ at time $t$, and that a fraction $\lambda \Delta t$ of firms with imbalance $x+\mu \Delta t$ at time $t$ adjusts before reaching $x$ because of a Calvo-type shock. From the derivation of Property 2 we know that the time-average of all possible cross-sections, $f_{A}(x)$, is equal to the ergodic distribution of an individual price setter. Let us calculate this average, as it is the concept we need to compute $\mathcal{A}$ and $\mathcal{F}$.

Setting $f(\cdot, t+\Delta t)=f(\cdot, t) \equiv h_{E}(\cdot)=f_{A}(\cdot)$ in the expression above, using a first-order 
Taylor expansion and letting $\Delta t \rightarrow 0$ leads to:

$$
f_{A}^{\prime}(x)=\alpha f_{A}(x)
$$

with $\alpha=\lambda / \mu$. Imposing that the integral of $f_{A}$ over the inaction range is one, then yields:

$$
f_{A}(x)=\frac{\alpha e^{\alpha(x+S-s)}}{e^{\alpha(S-s)}-1}, \quad s-S \leq x \leq 0
$$

Choosing the unit with which we measure time small enough so that the probability of two Poisson-shocks for the same firm in a given time-period is negligible, we have that the fraction of firms that adjust in one time period is:

$$
\text { Fraction of adjusters }=\lambda+(1-\lambda) F_{A}(s-S+\mu)
$$

where $F_{A}$ denotes the c.d.f. for $f_{A}$. The first term on the right hand side is the fraction of firms that adjust because of a Poisson shock. The second term considers, among those that did not receive such a shock, the fraction that adjusted because their state variable reached the trigger $s-S$. It follows that:

$$
\text { Fraction of adjusters } \cong \lambda+(1-\lambda) f_{A}(s-S) \mu \text {, }
$$

and from (10) we have that:

$$
\mathcal{A}^{\lambda} \cong \lambda\left(1+\frac{1-\lambda}{e^{\alpha(S-s)}-1}\right)
$$

Hence, since the fraction of agents that adjust before reaching the CS-trigger $s-S$ grows with $\lambda$, the frequency of adjustment index $\mathcal{A}^{\lambda}$ increases monotonically with $\lambda$.

For technical reasons, it is useful to turn to discrete time when calculating the priceflexibility index $\mathcal{F}$ once there is a Poisson term. We then have (as the impulse $\Delta m$ tends to zero):

$$
\frac{\Delta p^{\lambda}}{\Delta m} \cong \lambda+(1-\lambda) f_{A}(s-S)(S-s)
$$

The first term on the right hand side is the marginal price increase due to the monetary shock, for those firms with a Poisson-induced adjustment. The marginal response of these firms to the monetary shock is one-for-one. The second term is the additional contribution to inflation from firms that adjust because they reach the trigger barrier $s-S$. In contrast with the Poisson-induced adjusters, the response of these firms is $(S-s) / \Delta m$ times the 
shock that triggers their adjustment, and therefore much larger than one-to-one, so that $\lambda<\mathcal{F}^{\lambda}<1$ for $0<\lambda<1$. More precisely, substituting (10) in (14) yields

$$
\mathcal{F}^{\lambda} \equiv \frac{\Delta p^{\lambda}}{\Delta m}=\lambda+(1-\lambda) \frac{\alpha(S-s)}{e^{\alpha(S-s)}-1}
$$

Also note that $\mathcal{F}^{\lambda}$ does not vary monotonically with $\lambda$. It is one for $\lambda=0$ (the CS case) and again one for $\lambda=1$ (no micro frictions). It is decreasing for small values of $\lambda$ and increasing for larger values (see Figure 3).

Figure 3: $\mathcal{F}$ and $\mathcal{A}$ for a Caplin-Spulber model with Poisson Shocks

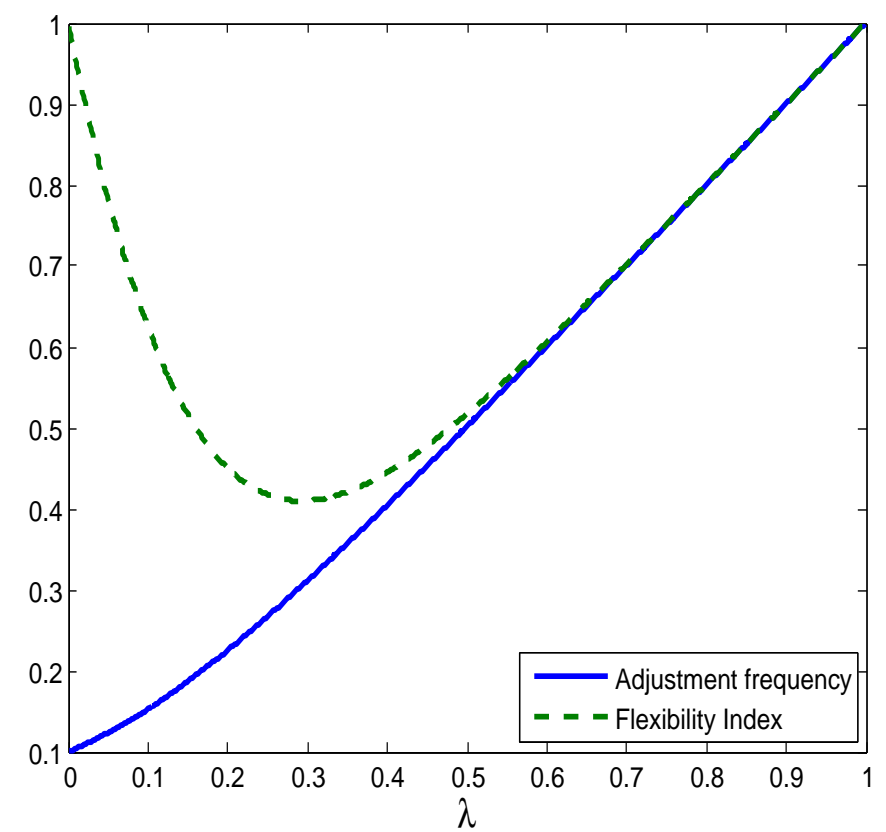

This simple example illustrates the complex connection between aggregate flexibility and the price adjustment frequency. Whether an increase in the frequency of price adjustments reduces price stickiness depends on how the increase in frequency affects the contribution of the intensive and extensive margins to the flexibility index $\mathcal{F}$.

The intensive margin refers to firms for which the monetary shock only affects firms behavior by increasing the price adjustment of those that would have adjusted anyway, with or without the monetary shock. These are the firms for which the Poisson shock triggers their adjustment, their contribution to $\mathcal{F}$ is the first term on the r.h.s. of (15). The extensive margin, by contrast, refers to firms whose adjustment is triggered by the marginal monetary shock (the impulse), their contribution to $\mathcal{F}$ is the second term on the r.h.s. of (15).

The contribution to $\mathcal{F}$ of the intensive margin increases with $\lambda$, this is obvious. What is 
less obvious is that the contribution of the extensive margin decreases, since the fraction of firms in the neighborhood of the trigger barrier $s-S, f(s-S)$, decreases. While the rate at which the intensive margin component of $\mathcal{F}$ increases with $\lambda$ is constant (and equal to one), the rate at which the extensive component decreases is large (in absolute value) for small values of $\lambda$ and small for larger values of $\lambda$. The reason for the latter is that the rate at which the fraction of firms in the neighborhood of the adjustment trigger $s-S$ decreases becomes smaller and smaller as $\lambda$ grows.

Taking stock, once we incorporate Poisson shocks into the CS environment, we have that, as expected, the flexibility and frequency of price adjustment indices move in the same direction for large values of $\lambda$ : more firms adjusting means that the aggregate price level is more responsive to shocks. Yet, as shown in Figure 3, which plots $\mathcal{F}^{\lambda}$ and $\mathcal{A}^{\lambda}$ as a function of $\lambda$ for a given $(s-S, 0]$ interval, for small values of $\lambda$ both indices move in opposite directions. The reason is that for small values of $\lambda$ the weakening of the extensive margin mechanism dominates over the standard positive relation between adjustment frequency and flexibility, explaining why $\mathcal{F}^{\lambda}$ decreases as $\lambda$ increases.

This example also helps motivate a point we develop in a more general context in the following section: The frequency of adjustment index is a lower bound for flexibility in $S s$ models, and this lower bound is achieved by the Calvo model. To see this, compare (13) and (15), and recall that our choice of time period ensures that $\mu<(S-s)$ (also recall that $\alpha=\lambda / \mu)$. It follows that, for $\lambda<1$ and $s$ finite,

$$
\mathcal{F}^{\lambda}>\mathcal{A}^{\lambda}
$$

The Calvo model is obtained by taking the limit as $s$ goes to minus infinity, in which case we have (for all $\lambda$ ):

$$
\lim _{s \longrightarrow-\infty} \mathcal{F}^{\lambda}=\lim _{s \longrightarrow-\infty} \mathcal{A}^{\lambda}=\lambda
$$

\section{The Extensive Margin Effect}

Let us generalize the model of the previous sections to consider a broader set of shocks and adjustment rules. These extensions are more easily implemented in discrete time. Shocks to the growth rate of money are i.i.d. with mean $\mu$ and variance $\sigma_{A}^{2}$, and firms experience idiosyncratic (productivity and demand) shocks $v_{i t}$ which are i.i.d. with zero mean and variance $\sigma_{I}^{2}$. No assumptions are made regarding the common distribution across aggregate (and idiosyncratic) shocks. These shocks are independent across agents and from the aggregate 
shock. With these assumptions, the target price follows the process:

$$
\Delta p_{i t}^{*}=\Delta m_{t}+v_{i t}
$$

With no further changes, and preserving the fixed cost of adjusting prices at the microeconomic level, this environment yields a two-sided Ss policy (see, e.g., Barro (1972)). We generalize it further and assume that there are i.i.d. idiosyncratic shocks to adjustment costs as well, drawn from a distribution $G(\omega)$. Integrating over all possible realizations of these adjustment costs, we obtain an adjustment hazard, $\Lambda(x)$, defined as the probability of adjusting prior to knowing the current adjustment cost draw, with ${ }^{7}$

$$
0 \leq \Lambda(x) \leq 1, \quad \forall x
$$

It follows that for non-degenerate distributions $G(\omega), \Lambda(x)$ is decreasing for $x<0$ and increasing for $x>0$ : the cost of deviating from the target price is increasing with respect to the distance from this price and therefore adjustment is more likely when $|x|$ is larger. This is the increasing hazard property (Caballero and Engel, 1993c).

Denoting by $f(x, t)$ the cross section of price imbalances immediately before adjustments take place at time $t$, we have:

$$
\Delta p_{t}=-\int x \Lambda(x) f(x, t) d x
$$

\subsection{A Basic Inequality}

Let $\Delta p_{0}\left(\Delta m^{d}\right)$ denote the average (over all possible cross-section distributions) inflation response to a monetary deviation of $\Delta m^{d}$ from its average growth rate. The first element of the impulse response function with respect to this shock is our flexibility index:

$$
\mathcal{F} \equiv \Delta p_{0}^{\prime}\left(\Delta m^{d}=0\right)
$$

\footnotetext{
${ }^{7}$ See Caballero and Engel (1999) for a detailed discussion of such a model, Dotsey, King and Wolman (1999) for an application to prices in a dynamic general equilibrium context, and Caballero and Engel (1993b) for an estimation of a generalized hazard model for prices.
} 
We have:

$$
\begin{aligned}
\Delta p_{0}\left(\Delta m^{d}\right) & =-\int x \Lambda(x) f_{A}\left(x+\Delta m^{d}\right) d x \\
& =-\int\left(x-\Delta m^{d}\right) \Lambda\left(x-\Delta m^{d}\right) f_{A}(x) d x
\end{aligned}
$$

Differentiating this expression with respect to $\Delta m^{d}$ and evaluating at $\Delta m^{d}=0$ yields:

$$
\mathcal{F}=\int \Lambda(x) f_{A}(x) d x+\int x \Lambda^{\prime}(x) f_{A}(x) d x
$$

Note that the first term on the right hand side corresponds to the adjustment frequency, $\mathcal{A}$. The second term, which we denote by $\mathcal{E}$, corresponds the contribution of the extensive margin (more on this shortly). Thus we can write:

$$
\mathcal{F}=\mathcal{A}+\mathcal{E}
$$

It follows that:

Property 4 (Flexibility and adjustment frequency are the same in the Calvo model)

In the Calvo model, where $\Lambda^{\prime}(x)=0$, we have that $\mathcal{E}=0$ and $\mathcal{F}^{\text {Calvo }}=\mathcal{A}^{\text {Calvo }}$. More importantly, it also follows that:

\section{Property 5 (The adjustment frequency is a lower bound for flexibility)}

In increasing hazard models we have $\mathcal{E}>0$, and therefore $\mathcal{F}>\mathcal{A}$.

Proof The increasing hazard property states that $\Lambda^{\prime}(x)>0$ for $x<0$ and $\Lambda^{\prime}(x)<0$ for $x>0$. It follows that $x \Lambda^{\prime}(x)>0$ for all $x$, and therefore $\mathcal{E}=\int x \Lambda^{\prime}(x) f(x) d x>0$ and $\mathcal{F}>\mathcal{A}^{8}$

The above results can be extended to the case with strategic complementarities along the lines of what we did in Section 2.5 (see Caballero and Engel, 2006). When the strategic complementarity parameter $a$ in (7) is positive, (18) becomes:

$$
\mathcal{F} \cong h(\mathcal{A}+\mathcal{E})
$$

\footnotetext{
${ }^{8}$ This result also holds if we work with the weaker concept of "increasing hazard property", according to which $\Lambda(x)$ satisfies this property if $\Lambda^{\prime}(x) \leq 0$ for $x \leq 0$ and $\Lambda^{\prime}(x) \geq 0$ for $x \geq 0$, with strict inequality on a set with positive measure (under the model's ergodic density).
} 
where $h(x)$ is a strictly increasing function. ${ }^{9}$ As before, a positive extensive margin $\mathcal{E}$ accounts for a larger average response of the price level to monetary shocks than suggested by the intensive margin $\mathcal{A}$.

\subsection{Extensions}

In this subsection we discuss two extensions of the above results. First, we consider the case of standard Ss policies, where the smoothness assumptions under which we derived (17) do not hold. Second, we show that the above inequality extends to the case of conditional impulse responses.

\subsubsection{Standard Ss Policies}

The above derivation assumes that $\Lambda(x)$ and $f_{A}(x)$ are continuous, which does not hold for standard Ss models, such as Barro (1972), Caplin and Spulber (1987), and other models where the distribution of adjustment costs is a mass point. ${ }^{10}$ As shown in the Appendix, when $x \Lambda(x) f(x)$ has jump-discontinuities at $x_{1}, x_{2}, x_{3}, \ldots$, expression (17) becomes:

$$
\mathcal{F}=\int \Lambda(x) f_{A}(x) d x+\int x \Lambda^{\prime}(x) f_{A}(x) d x+\sum_{k} x_{k}\left[\Lambda\left(x_{k}^{+}\right) f\left(x_{k}^{+}\right)-\Lambda\left(x_{k}^{-}\right) f\left(x_{k}^{-}\right)\right],
$$

where $f\left(x_{k}^{+}\right)$and $f\left(x_{k}^{-}\right)$denote the limit of $f(x)$ when $x$ approaches $x_{k}$ from the right (larger values than $x_{k}$ ) and left, respectively.

In particular, for a standard two-sided $S s$ policy, where firms' remain inactive when their price imbalance belongs to $[L, U]$, we have that the first, second and third terms on the right hand side of (19) are equal to $F_{A}(L)+\left(1-F_{A}(U)\right), 0$ and $|L| f_{A}(L)+U f_{A}(U)$, respectively, so that: ${ }^{11}$

$$
\mathcal{F}=F_{A}(L)+\left(1-F_{A}(U)\right)+|L| f_{A}(L)+U f_{A}(U),
$$

where $F_{A}$ denotes the c.d.f. corresponding to $f_{A}$.

It follows that for a standard two-sided $S s$ model we have:

$$
\begin{aligned}
\mathcal{A} & =F_{A}(L)+\left(1-F_{A}(U)\right), \\
\mathcal{E} & =|L| f_{A}(L)+U f_{A}(U) .
\end{aligned}
$$

\footnotetext{
${ }^{9} h(x)=(1-a) x /(1-a x)$.

${ }^{10}$ See Sheshinski and Weiss (1993) for a comprehensive volume on $S s$ models of pricing decisions.

${ }^{11}$ Bar-Ilan and Blinder (1992) derive a similar expression in the context of one-sided $S s$ rules.
} 


\subsubsection{Conditional Impulse Responses}

As we showed in Section 2, time variation in the cross-section distribution leads to priceflexibility indices that vary over time. In generalized $S s$ models, the cross section distribution of price imbalances is influenced by the sequence of monetary shocks hitting the economy. To quantify the extent to which the IRF fluctuates over time, we note that the expressions we derived above also apply to time-varying measures of price-adjustments and price-flexibility.

Denote by $f(x, t)$ the cross-section of price imbalances at time $t$, by $\mathcal{A}_{t}$ the fraction of firms adjusting their price in period $t$ and by $\mathcal{F}_{t}$ the first element of period $t$ 's impulse response function. We then have that:

$$
\mathcal{F}_{t}=A_{t}+\mathcal{E}_{t}
$$

with

$$
\mathcal{E}_{t} \equiv \int x \Lambda^{\prime}(x) f(x, t) d x
$$

That is, fluctuations in the IRF of generalized $S s$ models are driven by changes in the fraction of agents that adjust in any given time interval and in the extensive margin.

\section{Extensive Margin or Selection Effect?}

As mentioned earlier, the term $\mathcal{E}$ quantifies the importance of the extensive margin effect. That is, of the additional price increase resulting from the rise in the fraction of agents adjusting upwards and the fall in the fraction of agents adjusting downwards, both as a consequence of the additional monetary impulse. It contrasts with the intensive margin $\mathcal{A}$, which describes the additional price increase (or reduced price decrease) resulting from the larger adjustment (after the monetary impulse) of those that were going to adjust anyway. In the Calvo model, only the intensive margin is active, while in increasing hazard models both margins are strictly positive.

This extensive margin is a generalization of the effect of the number of agents reaching the trigger thresholds in Ss models discussed by Bar-Ilan and Blinder (1992). ${ }^{12}$ More recently, Golosov and Lucas (2006) have referred to the additional flexibility in $S s$ models as the selection effect, arguing that the agents that adjust their prices in response to a monetary shock are those that benefit the most from doing so. As we show below, the selection effect is not always an accurate description of the source of the additional flexibility present in $S s$ -

\footnotetext{
${ }^{12}$ For recent papers highlighting the role of the extensive margin see, for example, Klenow and Kryvtsov (2005) and Burstein and Hellwig (2006).
} 
type models. For this reason, we instead advocate using the extensive margin index defined above to characterize the additional responsiveness to monetary shocks in $S s$-type models.

In order to understand the mechanisms at work, we consider a firm with price imbalance $x$ and decompose its marginal response to a monetary shock, $\mathcal{F}(x)$, into the sum of the contributions of the intensive and extensive margins:

$$
\begin{aligned}
& \mathcal{A}(x)=\Lambda(x), \\
& \mathcal{E}(x)=x \Lambda^{\prime}(x)=\Lambda(x) \eta(x),
\end{aligned}
$$

where $\eta(x) \equiv x \Lambda^{\prime}(x) / \Lambda(x)$ denotes the elasticity of the adjustment hazard with respect to the price imbalance $x$.

An increasing hazard model is characterized by $\eta(x) \geq 0$, yet, as we show next, this does not always imply that $\mathcal{F}(x) \equiv \mathcal{A}(x)+\mathcal{E}(x)$ increases monotonically with $|x|$ (which is what a selection effect means).

\subsection{First Example}

Assume $\Lambda(x)$ belongs to the inverted normal family: ${ }^{13}$

$$
\Lambda(x)=1-e^{-\lambda_{2} x^{2}}
$$

with $\lambda_{2}>0$.

Figure 4 plots the intensive, extensive and total response as a function of the price imbalance $x$. As is the case with all increasing hazard models, $\mathcal{A}(x)$ is increasing in $|x|$. Some simple algebra shows that, for all $\lambda_{2}>0, \mathcal{E}(x)$ is increasing in $|x|$ for values of $x$ such that $\lambda_{2} x^{2}<1$, that is, values of $x$ with $\Lambda(x)<1-1 / e \cong 0.632$. For larger values of $|x|$, however, the extensive margin effect decreases as the magnitude of the price imbalance increases. It is therefore not surprising that in the range of price imbalances with sufficiently high probability of adjusting $\left(\Lambda(x)>1-e^{-3 / 2} \cong 0.78\right), \mathcal{F}(x)$ decreases monotonically with $|x| .^{14}$

For this family of adjustment hazards, the largest marginal price response to a monetary shock comes from firms with an ex-ante probability of adjusting slightly below 80\%. Firms with higher adjustment probabilities benefit more from changing their price but contribute less to the economy's marginal response to a monetary shock. It follows that the "selection

\footnotetext{
${ }^{13}$ See Caballero and Engel (1999) for an application of this family to investment.

${ }^{14}$ Both statements above follow from $\mathcal{A}(x)=1-e^{-\lambda_{2} x^{2}}, \mathcal{E}(x)=2 \lambda_{2} x^{2} e^{-\lambda_{2} x^{2}}, \mathcal{A}^{\prime}(x)=2 \lambda_{2} x e^{-\lambda_{2} x^{2}}$, $\mathcal{E}^{\prime}(x)=4 \lambda_{2} x\left(1-\lambda_{2} x^{2}\right) e^{-\lambda_{2} x^{2}}$ and $\mathcal{F}^{\prime}(x)=\lambda_{2} x\left(6-4 \lambda_{2} x^{2}\right) e^{-\lambda_{2} x^{2}}$.
} 
Figure 4: Generalized Ss Model where the Selection Effect does not hold

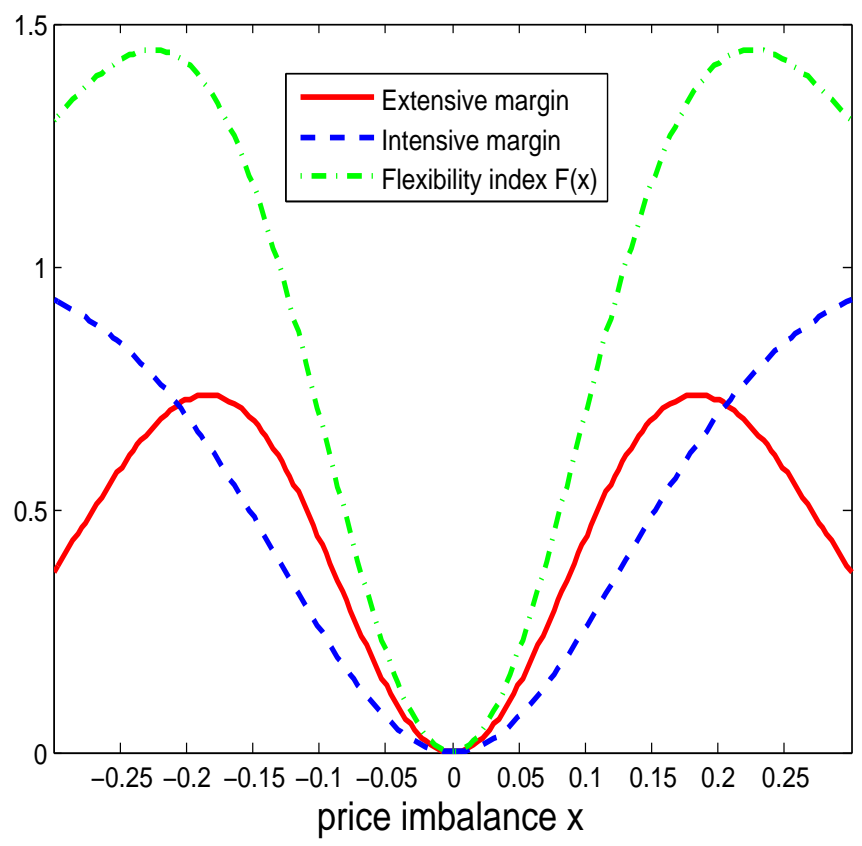

effect" does not provide the correct intuition underlying the larger price response to monetary shocks in this example.

\subsection{General Insight}

The insight of the preceding example is valid more broadly. In generalized $S s$ models, $\Lambda(x)$ attains its minimum value at $x=0$. Furthermore, often this minimum value is zero and $\Lambda(x)$ admits a quadratic approximation $\frac{1}{2} \Lambda^{\prime \prime}(0) x^{2}$ in a neighborhood of $x=0$, so that

$$
\eta(0)=\lim _{x \rightarrow 0} \frac{x \Lambda^{\prime}(x)}{\Lambda(x)}=\lim _{x \rightarrow 0} \frac{\Lambda^{\prime \prime}(0) x^{2}}{\frac{1}{2} \Lambda^{\prime \prime}(0) x^{2}}=2 .
$$

We also have that, in general, $\Lambda(x)$ approaches one as $|x|$ grows, so that $\Lambda^{\prime}(x)$, and therefore $\eta(x)$, approach zero. It follows that, as $|x|$ increases, $\eta(x)$ must decrease from a value of 2 at $x=0$ to values close to 0 when $|x|$ is large enough. ${ }^{15}$ Thus, two effects pull $\mathcal{F}(x)$ in opposite directions as $|x|$ increases. First, firms tolerate less well larger price imbalance and are more likely to adjust their price, that is, $\mathcal{A}(x)$ is increasing in $|x|$. If this were the only effect present, the selection effect would always hold. Yet there is a second effect: as $|x|$ grows

\footnotetext{
${ }^{15}$ Furthermore, in Caballero and Engel (2006) we show that if $\eta(x)$ remains close to 2 in a sufficiently large neighborhood of $x=0$, we have $\mathcal{E} \cong 2 \mathcal{A}$ and therefore $\mathcal{F} \cong 3 \mathcal{A}$.
} 
large enough, the elasticity $\eta(x)$ eventually is decreasing in $|x|$. This leads to a diminishing contribution of the extensive margin, $\Lambda(x) \eta(x)$, and points in the opposite direction. The selection effect provides the correct intuition only when the former effect dominates for all values of $x$. However the latter effect is likely to dominate for large enough $|x|$ in many $S$ s-type models, since $\mathcal{E}(x)$ is decreasing in this range of values while $\mathcal{A}(x)$ approaches a constant value of one.

\section{Property 6 (The Selection Effect and the Extensive Margin Effect)}

In generalized Ss models, the firms that contribute the most to the aggregate price level are not necessarily those that benefit the most from adjusting. This is due to the fact that the contribution of the extensive margin decreases with the size of the price imbalance when the latter is sufficiently large.

The above property also holds for standard $S s$ models in discrete time. Consider a two-sided $S s$ policy and the notation introduced when deriving (20). We then have:

$$
\frac{\Delta p_{i}}{\Delta m}(\Delta m, x)= \begin{cases}1, & x \leq L \\ 1+|x| / \Delta m, & L<x \leq L+\Delta m, \\ 0, & L+\Delta m<x<U, \\ 1+x / \Delta m, & U<x<U+\Delta m, \\ 1, & x \geq U+\Delta m .\end{cases}
$$

Firms with imbalances slightly above the trigger $L$ and slightly below the trigger $U$ are the ones that contribute most to the aggregate price level's marginal response to a monetary shock. The former, because the shock leads them to adjust their price upward when they would have remained inactive in the absence of the monetary shock. The latter, because the additional monetary expansion modifies their optimal choice from adjusting their price downward to not adjusting at all. In contrast, firms with larger imbalances $(x<<L$ or $x \gg U$ ) benefit more from adjusting but contribute less to the aggregate price response.

\subsection{Second Example}

In our first example the selection effect does not hold and yet $\mathcal{E}>0$. Next we provide an example where the selection effect is present and $\mathcal{E}=0$.

Aggregate shocks are drawn from a density with zero mean and bounded support, with a very small variance. Idiosyncratic shocks are drawn from the trimodal distribution depicted 
Figure 5: Density of idiosyncratic shocks

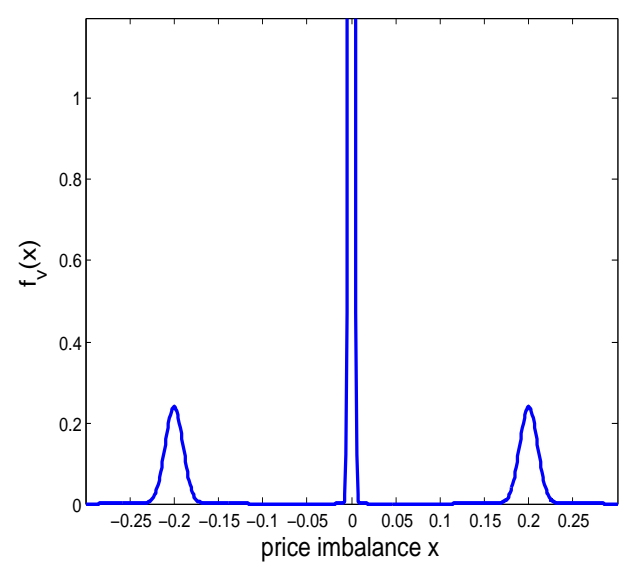

in Figure $5 .{ }^{16}$ With a high probability (0.8 in the figure), the idiosyncratic shock is zero while with a low probability ( 0.2 in the figure) it is the realization of one of two densities with bounded support that is far away from zero. These two densities only differ in their means, which have the same magnitude but opposite signs. It follows that idiosyncratic shocks are either large and positive, large and negative, or zero.

The cost of adjusting prices is a fixed fraction of firms profits, leading to a two sided Ss policy as in Barro (1972). The resulting ergodic density is shown in the solid line in Figure 6, which also shows the corresponding $S s$ bands (dashed line). As can be seen in this figure, there are no firms at the trigger barriers, so that $\mathcal{E}=0$ and $\mathcal{F}=\mathcal{A}$ in this case. The leptokurtic shape of idiosyncratic shocks implies that very few firms - in this simple and extreme example no firm at all-have their price imbalance in the region where marginal monetary shocks affect their decision to adjust. Firms either have large (absolute) price imbalances and adjust independent of the additional monetary shock, or have small deviations and will not adjust, with or without the marginal shock. Yet the selection effect is present since those firms that adjust are, indeed, those with largest imbalances and therefore those who most benefit from doing so, even though their marginal contribution to the price level is the same as that of adjusting firms in a Calvo model.

\footnotetext{
${ }^{16}$ This results in a leptokurtic distribution for idiosyncratic shocks, as in Midrigan (2006) and Gertler and Leahy (2006).
} 
Figure 6: Ergodic density and Ss bands

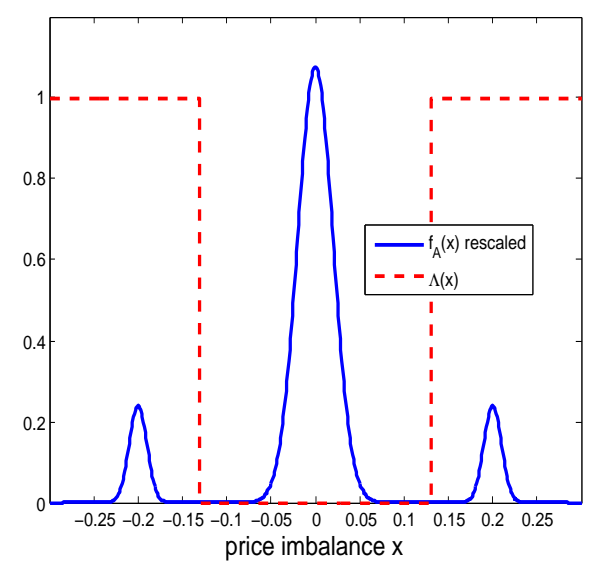

\subsection{Summary}

In summary, what matters for the additional flexibility of increasing hazard (and standard $S s)$ models is the increase in the fraction of agents adjusting in the direction of the impulse (and the decrease of agents adjusting in the opposite direction), caused by the impulse. Whether these changes in the decision to adjust come from agents that are near the tails or the target is a secondary factor.

Decomposing the flexibility index $\mathcal{F}$ into the sum of the contribution of the intensive and extensive margins, $\mathcal{A}$ and $\mathcal{E}$, provides a simple way of quantifying the additional price responsiveness present in $S s$-type models. The larger is $\mathcal{E}$, the larger is the contribution to the aggregate price level of time-variations in the fraction of firms adjusting.

We have provided an example where there is no selection effect and $\mathcal{E}>0$, and another example where the selection effect holds, yet $\mathcal{E}=0$. We conclude that selection is neither necessary nor sufficient to obtain an aggregate price level that, for a given frequency of price adjustments, responds more to monetary shocks than the corresponding Calvo model.

\section{Final Remarks}

The recent work of Golosov and Lucas (2006), Burstein (2006), Klenow and Krystow (2005), Midrigan (2006), Gertler and Leahy (2006), and others has rekindled the interest in menu cost type models. As with many microeconomic variables, the prices of goods and services are seldom adjusted continuously. However, the implications of microeconomic inaction for the 
stickiness of the aggregate price level is only gradually being understood, usually restricted to important but specific examples. In this article we have tried to take a step further in describing the conceptual issues involved in the connection between the frequency of price adjustments and aggregate price flexibility in generalized $S s$ models.

The contributions of this paper are pedagogical in nature. In particular, we clarified the source of the Caplin and Spulber (1987) neutrality result, which does not rely on the aggregation process but on the particulars of microeconomic adjustment in that model. We also showed that the reason for the additional flexibility of $S s$ over Calvo type models comes from an extensive margin effect. This effect is distinct from the so-called "selection effect," which is neither necessary nor sufficient for the additional flexibility.

The models we analyzed were intended to isolate a particular feature of discrete adjustment, not to offer an account of observed price stickiness. For that, the mechanisms we discussed need to be interacted with a variety of other sources of stickiness (Ball and Romer, 1990). On this account, the recent work by, e.g., Carvalho (2007), Gorodichenko (2006), Nakamura and Steinsson (2006b), Dotsey and King (2005) and Kleshchelski and Vincent (2007) seem particularly promising.

Moreover, in the main text we focused on increasing hazard models, but the results we derived also provide a hint on what kind of models are likely to generate substantial price rigidity: These are models with a negative extensive margin effect. Kehoe and Midrigan (2007) provide one such example, where the option to implement temporary sales effectively generates a decreasing hazard model for small deviations $x$. This feature is quantitatively important since the cross section distribution accumulates a large number of agents for small values of $x$, thus it is perfectly possible to generate an $\mathcal{E}<0$. The important message from our perspective, however, is that one can still understand the workings of their model with our framework.

Finally, it should be apparent that there is nothing specific to prices in our results. These are properties about contexts where lumpy microeconomic adjustment is prevalent, of which prices is just one application. At the microeconomic level, investment decisions, durable purchases, hiring and firing decisions, inventory accumulation, and many other important economic variables are lumpy in nature and hence fit the essentials of the model in this paper. $^{17}$

\footnotetext{
${ }^{17}$ For a recent application to investment see, e.g., Bachmann et al 2006. There we argue that in the US $\mathcal{F}$ is significantly less than one and time varying, with a substantial rise during the late 1990s.
} 


\section{References}

[1] Bachmann, R., R.J. Caballero and E.M.R.A. Engel (2006). "Lumpy Investment in Dynamic General Equilibrium," NBER Working Paper No. 12336.

[2] Ball, L. and D. Romer (1990). "Real Rigidities and the Non-Neutrality of Money," Review of Economic Studies, 57, 193-203.

[3] Bar-Ilan, A. and A. Blinder (1992). "Consumer Durables: Evidence on the Optimality of Usually Doing Nothing," Journal of Money, Credit and Banking, 24, 258-272.

[4] Barro, R.J. (1972). "A Theory of Monopolistic Price Adjustment" Review of Economic Studies 39, 17-26.

[5] Bils, M. and P.J. Klenov (2004). "Some Evidence on the Importance of Sticky Prices," Journal of Political Economy 112(5), 947-985.

[6] Burstein, A. (2006). "Inflation and Output Dynamics with State Dependent Pricing", Journal of Monetary Economics.

[7] Burstein, A. and M. Hellwig (2006). "Prices and Market Shares in a Menu-Cost Model," mimeo, UCLA.

[8] Caballero, R.J. and E.M.R.A. Engel (1991). "Dynamic $(S, s)$ Economies," Econometrica, 59(6), 1659-1686.

[9] (1993a). "Heterogeneity and Output Fluctuations in a Dynamic MenuCost Economy", Review of Economic Studies, 60, 95-119.

[10] (1993b). "Microeconomic Rigidities and Aggregate Price Dynamics", European Economic Review, 37 (4), 697-711.

[11] (1993c). "Microeconomic Adjustment Hazards and Aggregate Dynamics", Quarterly Journal of Economics, 108 (2), 359-383,

[12] (1999). "Explaining Investment Dynamics in US Manufacturing: A Generalized $(S, s)$ Approach," Econometrica 67(4), 741-782.

[13] (2006). "Price Stickiness in Ss Models: Basic Properties," MIT and Yale mimeo, October 2006

[14] Calvo, G. (1983). "Staggered Prices in a Utility Maximizing Framework" Journal of Monetary Economics, 12, 383-398

[15] Caplin, A. and D. Spulber (1987). "Menu Costs and the Neutrality of Money," Quarterly Journal of Economics, 102, 703-726. 
[16] Carvalho, C. (2006). "Heterogeneity in Price Stickiness and the Real Effects of Monetary Shocks," Frontiers of Macroeconomics, 2(1), Article 1.

[17] Dhyne, E., L.J. Alvarez, H.L. Bhan, G. Veronese, D. Dias, J.. hoffmann, N. Jonker, P. Lunnemann, F. Rumler and J. Vilmunen (2006). "Price Setting in the Euro Area and the United States: Some Facts ¿From Individual Consumer Price Data," Journal of Economic Perspectives, 20(2), 171-192.

[18] Dotsey, M., and R.G. King (2005). "Implications of state-dependent pricing for dynamic macroeconomic models," Journal of Monetary Economics, 52, 213-242.

[19] Dotsey, M., R.G. King, and A.L. Wolman (1999). "State Dependet Pricing and the General Equilibrium Dynamics of Money and Output" Quarterly Journal of Economics, 114, 655-690

[20] Fabiani, S., M. Druant, I. Hernando, C. Kwapil, B. Landau, C. Loupias, F. Martins, T. Matha, R. Sabbatini, H. Stahl, and A. Stokman (2006). "The Pricing Behavior of Firms in the Euro Area: New Survey Evidence," International Journal of Central Banking, 2 (3), September, 3-47.

[21] Gertler, M. and J. Leahy (2006). "A Phillips Curve with an Ss Foundation," NBER Working Papers 11971.

[22] Golosov, M. and R.E. Lucas (2006). "Menu Costs and the Phillips Curve," MIT, mimeo.

[23] Kehoe, P. and V. Midrigan (2007). "Sales, Clustering of Price Changes, and the Real Effects of Monetary Policy," mimeo.

[24] Klenow, P.J. and O. Kryvtsov (2005). "State-Dependent or Time-Dependent Pricing: Does It Matter for Recent U.S. Inflation," NBER Working Paper No. 11043.

[25] Kleshchelski, I. and N. Vincent (2007). "Market Share and Price Rigidity," Northwestern, mimeo.

[26] Midrigan, V. (2006). "Menu Costs, Multi-product Firms, and Aggregate Fluctuations." Mimeo.

[27] Nakamura, E. and J. Steinsson (2006a). "Five Facts About Prices: A Reevaluation of Menu Cost Models," mimeo, Harvard University.

[28] Nakamura, E. and J. Steinsson (2006b). "Monetary Non-Neutrality in a Multi-Sector Menu Cost Model," mimeo, Harvard University.

[29] Sheshinski, E. and Y. Weiss (1993). Optimum Pricing, Inflation, and the Cost of Price Adjustment, (eds.). MIT Press.

[30] Stokey, N. (2002). Brownian Models in Economics. Manuscript.

[31] Walters, P. (1982). An Introduction to Ergodic Theory, Springer-Verlag. 


\section{Appendix: Formal Proofs}

Proposition A 1 (The Case with Discontinuities) Denote by $\Lambda(x)$ the adjustment hazard, $f(x)$ the cross-section of price imbalances, $\mathcal{F}$ the flexibility index and $\mathcal{A}$ the fraction of adjusters. Assume that $x \Lambda(x) f(x)$ is discontinuous at $x_{1}<x_{2}<\ldots<x_{n}$, with a jump $D_{k}$ at $x_{k}$ :

$$
D_{k} \equiv x_{k}\left[\Lambda\left(x_{k}^{+}\right) f\left(x_{k}^{+}\right)-\Lambda\left(x_{k}^{-}\right) f\left(x_{k}^{-}\right)\right],
$$

where $f\left(x_{k}^{+}\right)$and $f\left(x_{k}^{-}\right)$denote the limit of $f(x)$ when $x$ approaches $x_{k}$ from the right (larger values than $x_{k}$ ) and left, respectively. Also assume $\lim _{x \rightarrow \pm \infty} x \Lambda(x) f(x)=0$. We then have:

$$
\begin{aligned}
& \mathcal{A}=\int_{-\infty}^{\infty} \Lambda(x) f(x) d x \\
& \mathcal{F}=\mathcal{A}+\sum_{k=1}^{n} D_{k}+\int_{-\infty}^{\infty} x \Lambda^{\prime}(x) f(x) d x .
\end{aligned}
$$

Proof The expression for $\mathcal{A}$ is straightforward. To derive the expression for $\mathcal{F}$, denote $A_{i}=\left(x_{i-1}, x_{i}\right), i=1,2 \ldots, n+1$; with $x_{0} \equiv-\infty$ and $x_{n+1} \equiv \infty$. Denoting by $\Delta p_{0}(v)$ the change in the price level due to a monetary shock $v$ we have:

$$
\Delta p_{0}(v)=-\int(x-v) \Lambda(x-v) f(x) d x=-\sum_{i=1}^{n+1} \int_{x_{i-1}+v}^{x_{i}+v}(x-v) \Lambda(x-v) f(x) d x .
$$

A basic result from calculus (Leibniz's formula) states that if $a(v), b(v)$ and $g(x, v)$ are differentiable and

$$
G(v) \equiv \int_{a(v)}^{b(v)} g(x, v) d x
$$

then

$$
G^{\prime}(v)=g(b(v), v) b^{\prime}(v)-g(a(v), v) a^{\prime}(v)+\int_{a(v)}^{b(v)} \frac{\partial g}{\partial v}(x, v) d x .
$$

Applying this result to calculate the $n+1$ integrals in the sum on the r.h.s. of (24), differentiating w.r.t. $v$ and evaluating at $v=0$ :

$$
\mathcal{F}=-\sum_{i=1}^{n+1}\left\{x_{i} \Lambda\left(x_{i}^{-}\right) f\left(x_{i}^{-}\right)-x_{i-1} \Lambda\left(x_{i-1}^{+}\right) f\left(x_{i-1}^{+}\right)\right\}+\sum_{i=1}^{n+1} \int_{x_{i-1}}^{x_{i}}\left[\Lambda(x)+x \Lambda^{\prime}(x)\right] f(x) d x .
$$

Since we assumed $\lim _{x \rightarrow \pm \infty} x \Lambda(x) f(x)=0$, the first sum on the r.h.s. of (25) is equal to the sum of the jumps of $x \Lambda(x) f(x)$. The expression for $\mathcal{F}$ now follows. 
Corollary A 1 If the assumptions of Proposition 1 hold and $\Lambda\left(x_{k}^{+}\right) f\left(x_{k}^{+}\right)-\Lambda\left(x_{k}^{-}\right) f\left(x_{k}^{-}\right)$is negative when $x_{k}<0$ and positive when $x_{k}>0$, as is the case for most $S$ s-type models, then $\mathcal{F} \geq \mathcal{A}$.

Corollary A 2 (Caplin and Spulber) To obtain a discrete-time approximation to the Caplin and Spulber model, we consider $f(x)$ uniform on $(s-S-\mu,-\mu]$, since the relevant crosssection is the one after the aggregate shock (of size $\mu$ ), immediately before adjustments take place. We also assume $\Lambda(x)=0$ if $x \in(s-S, 0)$ and $\Lambda(x)=1$ otherwise. Proposition $A 1$ then implies that:

$$
\mathcal{F}^{\mathrm{CS}}=1+\frac{\mu}{S-s}
$$

Of course, a uniform distribution on the inaction range is only an approximation to the ergodic density in discrete time. This explains why we obtain even more flexibility than in the continuous time case.

If we adapt (15) to discrete time, we have:

$$
\mathcal{F}^{\lambda}=\lambda+(1-\lambda) \frac{\alpha(S-s+\mu)}{e^{\alpha(S-s)}-1},
$$

and we obtain (26) letting $\lambda \rightarrow 0$. 